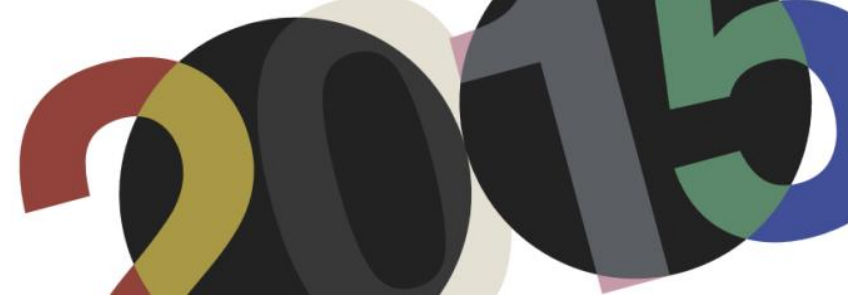

DOI: http://dx.doi.org/10.4995/LC2015.2015.652

\title{
Concevoir aujourd'hui un film sur la villa Savoye
}

\author{
A. Dyephart
}

Ecole Nationale Supérieure d'Architecture de Paris-Belleville

Résumé: Si de nombreuses images de la villa Savoye existent, par contre peu de films sont centrés sur elle. Je souhaite présenter ici un projet de film documentaire que je développe depuis 2012. Sur cette villa considérée comme une icône de l'architecture moderne, et déjà tant étudiée, je propose un regard un peu décalé, nourri à la fois par ma formation d'architecte et mon expérience de réalisatrice. J'aborde en particulier la question de l'impact de cette architecture; comment est-elle perçue au XXIème siècle, en quoi peut-elle influer sur notre façon d'envisager l'espace?

Je développerai un aspect de ma démarche qui touche au rapport entre architecture et cinéma: comment montrer la villa par un film? autrement dit comment traduire ce qu'elle représente par des images animées, des sons, mais aussi des 'évènements' qui dévoilent les contrastes de lumière et d'ambiances? De quoi cette architecture est-elle le récit? Ou, comme le proclame une des questions-slogans de Bernard Tschumi : "Et si l'architecture était autant une question de mouvement et d'évènement que d'espace?"1.

Resumen: Si existen numerosas imágenes de la Villa Savoye, escasos son, en cambio, los films que traten de ella. Deseo presentar aquí un proyecto de film documenta, sobre el que estoy trabajando desde el año 2012. Propongo una vision un poco desfasada, nutrida al mismo tiempo por mi formación de arquitecta y mi experiencia de cineasta, sobre esta obra ya tan estudiada, icono de la arquitectura moderna. Abordo especialmente la cuestión del impacto de esta arquitectura; ¿cómo es percibida en el siglo XXI?, ¿qué influencia puede tener en nuestra manera de considerar el espacio?

Desarrollaré un aspecto de mi trabajo que trata de la relación entre la arquitectura y el cine: como mostrar la Villa Savoye en un film? Es decir, como traducir lo que ella representa a través de imágenes animadas, sonido y "acontecimientos" que rebelen los contrastes de luces y de ambientes? ¿ De qué da cuenta esta arquitectura? $O$, como lo proclama una de las preguntas-eslogan de Bernard Tschumi: “'Y si la arquitectura fuera tanto cuestión de movimiento y de acontecimiento como de espacio?"

Mots-clés: villa Savoye ; film documentaire ; transmission ; création ; recherche formelle.

Palabras clave: villa Savoye, película documental, transmisión, creación, investigación formal.

\section{Questions de points de vue}
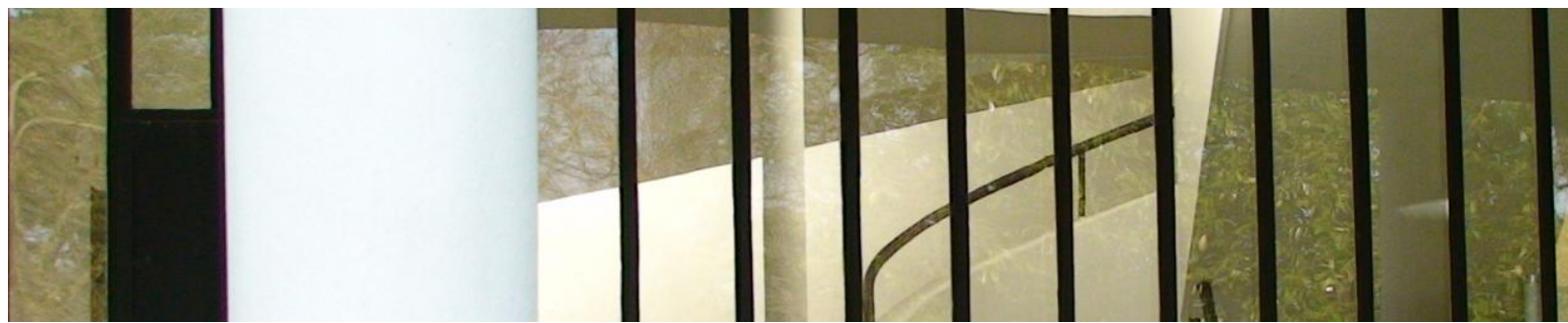

1. Photographie de repérage, hall de la villa Savoye (A.D. 2013). ( F FLC

\footnotetext{
${ }^{1}$ Tschumi, Bernard : Exposition Rétrospective Centre Georges Pompidou 2014.
} 


\subsection{Réception de l'architecture, fenêtre télévisuelle}

Architecte DPLG, je me suis formée à la réalisation de films à la fin des années 90 . Je voulais m'approprier cet outil visuel qui présente l'avantage de concilier des approches plastiques, sonores, textuelles et temporelles, afin de participer à la sensibilisation du public à l'architecture. Le documentaire de création était alors une forme en plein développement, porteuse d'innovation. J'ai également entamé des recherches sur la représentation de l'architecture contemporaine, sur les chaînes publiques françaises de télévision, depuis les années 1960 jusqu'aux années 1990. J'ai mené cette étude dans le cadre d'un DEA puis d'un contrat avec le Ministère de la Culture $^{2}$, motivée par plusieurs constats. Depuis la fin de mes études d'architecture, j'étais frappée par le fossé séparant souvent architectes et habitants, et j'étais convaincue que la discipline architecturale gagnerait à être plus largement connue. J'étais tout aussi intriguée par une présence parcimonieuse et assez formatée de l'architecture sur ce media populaire. Je croyais encore à la mission éducative de la télévision et je savais qu'en France le documentaire était financé principalement par les chaînes publiques. Je voulais donc analyser les films sur l'architecture qui avaient été diffusés, interroger les fréquences de ces diffusions et leurs formes, leur évolution dans le temps, espérant comprendre ce phénomène.

Cette recherche a confirmé le désintérêt des programmateurs de chaînes françaises pour le sujet. Les années 1980 avaient pourtant vu se développer des tentatives pour montrer l'architecture d'une manière vivante, dans une diversité de durées, de formes, en osant des approches originales parfois transdisciplinaires. Mais cette embellie fut de courte durée. Depuis les années 2000, on constate même de plus en plus une sorte de rejet. Les programmateurs de chaîne considèrent l'architecture comme un sujet élitiste, qui ne peut intéresser le public. Aussi, quand ils acceptent de l'aborder, ils préfèrent le portrait de star ou l'actualité médiatique à l'exploration d'une oeuvre ou d'un thème architecturaux. En dehors de la collection de monographies 'Architectures', d'ailleurs très bien faite mais assez fermée, diffusée par la chaîne Arte, point de salut ${ }^{3}$. Cette fermeture est-elle spécifique au monde de la télévision, ou fait-elle partie d'une incompréhension partagée par d'autres acteurs de la société, regardant l'architecture comme 'un monde à part' ne les concernant pas ? La question est à creuser.

Pour revenir à l'image animée j'ai constaté, d'abord comme cinéphile puis dans mes expériences de réalisatrice, les liens intimes existant entre architecture et cinéma. J'ai conçu mon tout premier court-métrage de manière intuitive, en dessinant un story-board, et j'avais l'impression de renouer avec ma pratique du projet architectural. Les relations architecture-cinéma sont d'ailleurs sporadiquement le prétexte de débats ou colloques. Ainsi en 1998, une série de rencontres intitulées 'un architecte, un cinéaste' ont été programmées au Centre Pompidou, présentées par Hubert Damish ${ }^{4}$. Ces dialogues révélaient les nombreux croisements tissés entre ces deux disciplines, architecture et cinéma, des rapports sans doute profonds mais parfois difficiles à cerner. On y sentait aussi l'intérêt réciproque entre architectes et cinéastes. Parmi ces derniers, cependant, plusieurs affirmaient la difficulté à filmer l'architecture.

\footnotetext{
${ }^{2}$ Existe-t-il un espace public télévisuel pour l'architecture contemporaine? DEA Cinéma, Télévision, Audiovisuel, Paris1 Panthéon-Sorbonne, 1999. Complété par une recherche pour la DAPA, Ministère de la Culture, Paris 2001 : Panorama des sources documentaires audiovisuelles sur le thème de l'architecture et la ville diffusées par les chaînes publiques hertziennes. Études signées de mon nom d'état civil : Annick Pancher.

3 "En dehors de la collection, il arrive que nous faisions un projet isolé, mais il faut vraiment qu'il soit particulier (...) La collection Architectures, c'est assez pour parler d'architecture." Thierry Garrel, Responsable de l'Unité de Programmes Documentaires ARTE, 1999, entretien mené dans le cadre du DEA déjà cité Existe-t-il un espace public télévisuel pour l'architecture contemporaine?

${ }^{4}$ Un architecte - un cinéaste: Cycle de débats animés par Hubert Damisch, dont les rencontres entre Alain Fleischer et Bernard Tschumi, Pierre Riboulet et Jean-Louis Comolli, Henri Gaudin et Cédric Klapish, Robert Kramer et Rem Koolhaas, au Centre Georges Pompidou, 1998.
} 
Cette résistance au film explique-t-elle la rareté des documentaires sur l'architecture ? Est-elle liée à la nature de l'architecture, ou à une certaine rigidité dans le regard porté sur elle ? Lors des rencontres évoquées plus tôt, Jean-Louis Comolli dialoguait avec Pierre Riboulet, pour qui il a réalisé le très beau documentaire Naissance d'un hôpital ${ }^{5}$. Il affirmait "Ce qui est gênant dans la relation du cinéma à l'architecture, c'est qu'elle se présente comme quelque chose de visible, c'est le piège, le leurre (...) le cinéma aura peut-être le désir de faire apparaître à l'intérieur de l'architecture ce qui est dissimulé." En me remémorant certains de ces échanges, je suis convaincue qu'une véritable recherche formelle s'impose pour filmer l'architecture.

Pour recentrer mon propos, donc, je pars de ce constat : l'architecture n'a pas 'bonne presse' chez les diffuseurs. Peur d'un sujet mal connu? incompréhension? Leur attitude pose la question de la réception de l'architecture. Et ce désintérêt décourage les initiatives de réalisateurs et de producteurs dans ce domaine. Il faut donc pour développer ce type de projet une vraie conviction. La mienne est que l'innovation dans l'écriture filmique a un rôle à jouer dans cette transmission de l'architecture. Comment mieux en parler qu'à travers un projet concret?

\subsection{La villa Savoye, images}

La villa Savoye s'est imposée à moi pour ce projet de film. Peut-être parce que dès mes études, en décryptant ses plans et photos, j'y ai senti une pointe de mystère. Et que ma première visite à la villa est nimbée de cette lumière particulière qui enveloppe une première journée sous la neige, un jour de janvier, alors que jeune architecte diplômée j'entre dans ce parc, je marche au milieu des arbres et découvre une silhouette à la fois frêle et assuree, blanche sur fond blanc, autour de laquelle papillonnent les silhouettes sombres des autres visiteurs. Émotion forte qui s'enrichit au fil de la découverte.

La villa Savoye est aussi un lieu de contradictions. Monumentale dans son allure et pourtant accueillante, à l'échelle de l'homme; généreuse par la qualité et la variété de ses espaces, et pourtant austère, sobre par ses matériaux ; villa 'blanche' faisant chanter des couleurs. Jalon dans l'oeuvre de Le Corbusier, elle représente une synthèse de ses expérimentations sur l'espace domestique avant de construire à plus grande échelle ; mais elle fut très peu habitée. Enfin la villa saisit par un mélange de rigueur et de poésie, elle exprime une certaine idée de l'architecture, et de la vie. À la fois photogénique et invitant au mouvement, par la promenade architecturale qu'elle incarne, elle donne envie de la filmer. Qu'est-ce qu'un film ? Une capture du réel par l'image animée : la possibilité d'une continuité, un travail de cadrage, une attention à la lumière et au mouvement, une succession de séquences, la recherche d'un rythme. Ceci est un raccourci, bien sûr, mais au-delà des cinq principes d'une architecture moderne, les mêmes termes pourraient être utilisés pour évoquer les villas dites 'blanches' des années 1920, en particulier la villa Savoye. Son caractère emblématique, la singularité de son destin et la pérennité de son empreinte en font un personnage idéal pour un film qui souhaite inviter un public de néophytes à mieux appréhender l'architecture.

Que montrent les videos amateur captées par des visiteurs et proposées sur des sites publics tels 'youtube' ou 'dailymotion'? Le visiteur-filmeur nous embarque dans son parcours, avec ses à-coups, ses surprises, mais aussi ses difficultés à restituer la magie de cette promenade. Pourquoi ? Parce qu'une image embarquée sur un itinéraire figé (presque toujours le même dans toutes les vidéos) réduit le champ du perceptible, laissant à peine au regard le temps de l'adaptation aux différentes échelles et ambiances, la possibilité d'un recul, ne lisant que les évidences. Surtout il manque une structure, un récit, un propos : ce qu'un film construit peut tenter de restituer, mais non une simple captation.

\footnotetext{
${ }^{5}$ Naissance d'un hôpital : documentaire de Jean-Louis Comolli, 1h07, 1992, tiré du livre Naissance d'un hôpital de Pierre Riboulet, Editions Plon, 1988.
} 
Le court métrage Architecture d'aujourd'hui ${ }^{6}$, réalisé dès 1931 par Pierre Chenal, montre la villa Church, la villa Stein et la villa Savoye à peine terminée. Les images sont émouvantes par le grain et les discontinuités du mouvement, l'allure et les vêtements des personnages, qui suggèrent fortement leur époque. Elles mettent en avant les espaces extérieurs, et transmettent la dynamique si importante pour Le Corbusier. La brève séquence sur la villa Savoye, par exemple, met en scène une femme qui gravit la rampe extérieure.
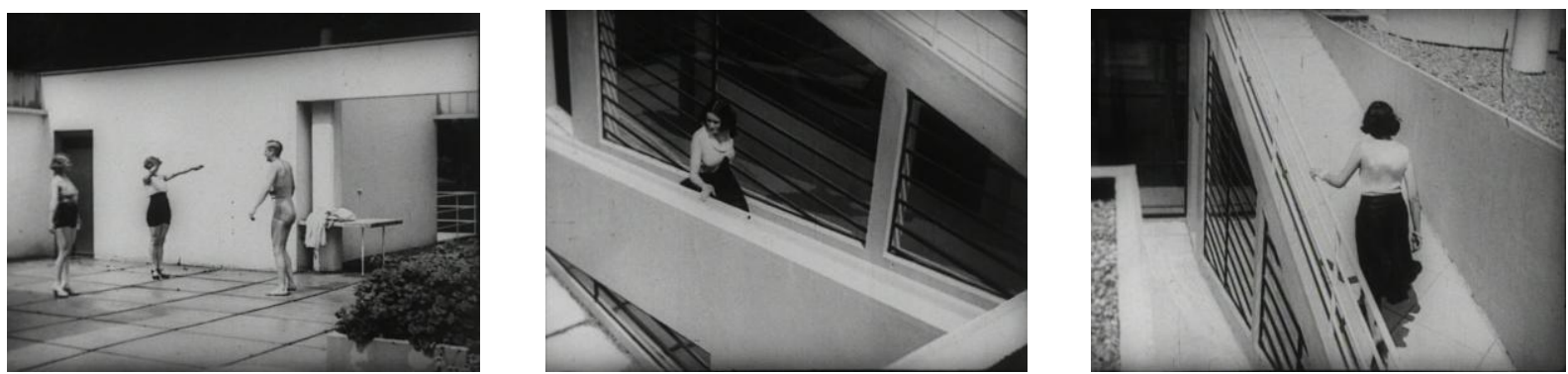

2. - 3. - 4. Photogrammes du film ‘Architectures d'aujourd'hui’ de Pierre Chenal, 1931. @ FLC

Le seul film à ce jour entièrement consacré à la villa Savoye, Le Corbusier - villa Savoye ${ }^{7}$, est écrit et présenté par l'historien Tim Benton en 1973. C'est un documentaire didactique, diffusé par la BBC pour l'Open University. Tim Benton y est un guide qui parcourt la villa pour décrypter en quoi elle est une 'machine à habiter', et pointe ses particularités formelles et fonctionnelles.

Mon projet de film propose un éclairage différent, en interrogeant la villa Savoye sur sa vivacité, ce qu'elle nous transmet aujourd'hui, et l'esprit de création.

\subsection{Dialogue entre film et architecture}

Cette communication vise à témoigner de la démarche suivie pour concevoir un tel film. Elle s'appuie sur mes interrogations quant à la manière de transmettre les qualités d'une oeuvre architecturale. Ce travail montre que l'écriture d'un documentaire s'établit sur un réseau de relations avec son sujet, dont découle une forme. Elle peut s'assimiler à un processus de recherche.

Parce qu'il permet de parcourir l'espace, de le découper, de le simuler, d'y inclure des personnes et des actions dans une continuité, le film est un véritable outil de médiation pour l'architecture. Il permet une approche plus synthétique qu'un texte ou une image fixe, il les complète et invite à la visite. Le film, fiction ou documentaire, explore et reconstruit tout à la fois des lieux, dans une certaine durée, par le choix des vues et le rythme du montage.

Un film documentaire se distingue du reportage en ce qu'il est écrit, composé, et porté par des intentions d'auteur. Il ne s'agit pas d'un travail journalistique, se voulant objectif. Un film documentaire apporte des informations sur un sujet, mais il est aussi l'expression d'un regard personnel sur ce dernier, le résultat d'une démarche subjective. C'est pourquoi le documentaire peut prendre des formes très variées, montage d'archives, suite d'interviews, enquête, reconstitutions, immersion dans un milieu, création poétique, etc. L'histoire du documentaire montre aussi qu'il a toujours joué avec les limites de la fiction. Comme l'exprime autrement le réalisateur et essayiste Jean-Louis Comolli "Tout est récit au cinéma et un film documentaire est un récit autant qu'un film de fiction" ${ }^{8}$. Le documentaire, par les images et les situations filmées autant que par des discours, questionne son sujet. Il vise à en approfondir la compréhension, et dans ce but recompose, interprète, met à

\footnotetext{
${ }^{6}$ Architecture d'aujourd'hui, film 16mm, N\&B, muet, réalisé par Pierre Chenal, 10min, 1931.

${ }^{7}$ Le Corbusier-Villa Savoye, film 16mm réalisé par Tim Benton, couleur, sonore, 24min., 1973.

${ }^{8}$ Rencontre avec Pierre Riboulet, dans le cycle un architecte-un cinéaste, déjà citée.
} 
distance. A condition de mener une véritable recherche de points de vue pertinents, et d'offrir au spectateur cet écart nécessaire pour qu'il trouve sa propre lecture, le documentaire sur l'architecture permet de transformer le regard, le décaler, le préciser, pour déployer notre perception et notre connaissance du sujet. Il devient outil de connaissance.

Je ne résiste pas à la tentation du clin d'oeil. Dès les années 1920 Le Corbusier souhaite que son architecture soit filmée ; il sollicite en particulier Pierre Chenal pour réaliser les films Bâtir (1928) et Architecture d'aujourd'hui (1931) déjà cité, et Jean Epstein pour Les bâtisseurs (1931). Il utilise l'accumulation de photographies dans une conférence de 1924 "censée produire un défilement cinématographique"9 pour montrer la villa La Roche, comme le rapporte Clotilde Simond. Il a enregistré lui-même quelques bobines de film avec une caméra $16 \mathrm{~mm}$, en 1936 et 1937, vues de son voyage en Amérique latine, d'éléments de nature, de quelques intérieurs. "Le brutalisme des cadrages et les fréquentes contre-plongées rapprochent le cinéma de Le Corbusier de celui de deux cinéastes qui ont intéressé le maître : l'un très connu dès les années 1920, Sergueï M.Eisenstein, et l'autre moins célèbre, Maya Deren, dont il fera l'éloge dans les années 1940"10 note Claude Prelorenzo dans un article où il explore ces images animées, alternant avec des photographies prises avec la même caméra. Ces courtes séquences filmées sont restées longtemps confidentielles, mais confirment un véritable intérêt pour une technique qui permet de représenter un regard en mouvement. Ses jugements sur le cinéma évolueront au cours du temps. Il privilégie d'abord le documentaire, comme le souligne Arnaud François dans un article de $1996{ }^{11}$ : "Voilà pourquoi il fit faire tant de films sur ses architectures, pour que le public puisse en saisir la vivacité. Les documentaires qu'il réalisa sur ses constructions avaient deux objectifs : d'une part présenter son oeuvre, et d'autre part mettre en avant le regard subjectif, l'intensification de la vision qui structure son architecture." Recherchant l'efficacité des films pour promouvoir son architecture, il s'ouvre aussi à une utilisation de la mise en scène. Ainsi il incarne son propre rôle dans La vie commence demain ${ }^{12}$, où il dialogue avec l'acteur JeanPierre Aumont qui joue le naïf sur le chantier de la Cité Radieuse de Marseille. Dans les années 1950 il aura le projet d'une véritable fiction au même endroit, avec Camus comme scénariste ${ }^{13}$, projet qui ne semble pas s'être concrétisé.

\section{Filmer la villa Savoye, explorer}

"Suggérer, c'est créer. Décrire, c'est détruire".

Cette phrase de Doisneau m'accompagne depuis longtemps et imprègne ce projet.

\subsection{Le point de vue du film : de l'importance des personnages}

Pour ce projet de film sur la villa Savoye, ma démarche s'appuie dans son intention sur la formule exprimée très tôt par le héros de ce colloque : 'une architecture pour émouvoir' ${ }^{14}$. Jeune étudiante, découvrant en même temps

\footnotetext{
${ }^{9}$ Simond, Clotilde : Cinéma, architecture, arts technologiques : pour quel être ensemble ?, in In situ-de visu-in motu, Infolio éditions, 2014.

${ }^{10}$ Prelorenzo, Claude : Quand Corbu faisait son cinéma, in Le Visiteur $\mathrm{n}^{\circ}$ 17, 2011.

${ }^{11}$ François , Arnaud: La cinématographie de l'oeuvre de Le Corbusier, in Cinémathèque, printemps 1996.

${ }^{12}$ La vie commence demain : film $35 \mathrm{~mm}$ réalisé par Nicole Védrès et Jean-Pierre Aumont, $\mathrm{N} \& \mathrm{~B}$, sonore, commentaires écrits par Le Corbusier, 96min, 1949.

${ }^{13}$ François, Arnaud : article cité ci-dessus.

${ }^{14}$ Le Corbusier : "L'ARCHITECTURE est un fait d'art, un phénomène d'émotion, en dehors des questions de construction, au delà. La Construction, C'EST POUR FAIRE TENIR; l'Architecture, C'EST POUR EMOUVOIR.” in Vers une architecture, 1923.
} 
les ordres de l'architecture antique et les élans de l'architecture moderne, je me souviens avoir été intriguée par le mélange de liberté et de rigueur que je lisais dans les dessins de Le Corbusier. Les photos, les plans de ses constructions, et surtout ses croquis perspectifs avaient sur moi le pouvoir du rêve, de la suggestion. Ils me donnaient accès à une façon 'autre' d'envisager notre mode de vie.

J'ai retrouvé en visitant la villa Savoye cette impression d'un lieu permettant d'accéder à d'autres possibles, d'agrandir mon quotidien. J'y vois une force mystérieuse, parce qu'elle cristallise un moment fort d'une pensée architecturale, expérimente des principes encore efficaces aujourd'hui. Les infiltrations d'eau, puis bien plus gravement la guerre de 1940 ont causé des dommages qui ont bouleversé l'usage de la villa Savoye, jusqu'à ce qu'elle soit menacée de destruction à la fin des années 50. Vous en connaissez l'histoire. Devenue monument historique et restaurée laborieusement, elle ne contient aucune trace de ses habitants, ce qui provoque chez certains visiteurs une impression de vide, voire de froideur.

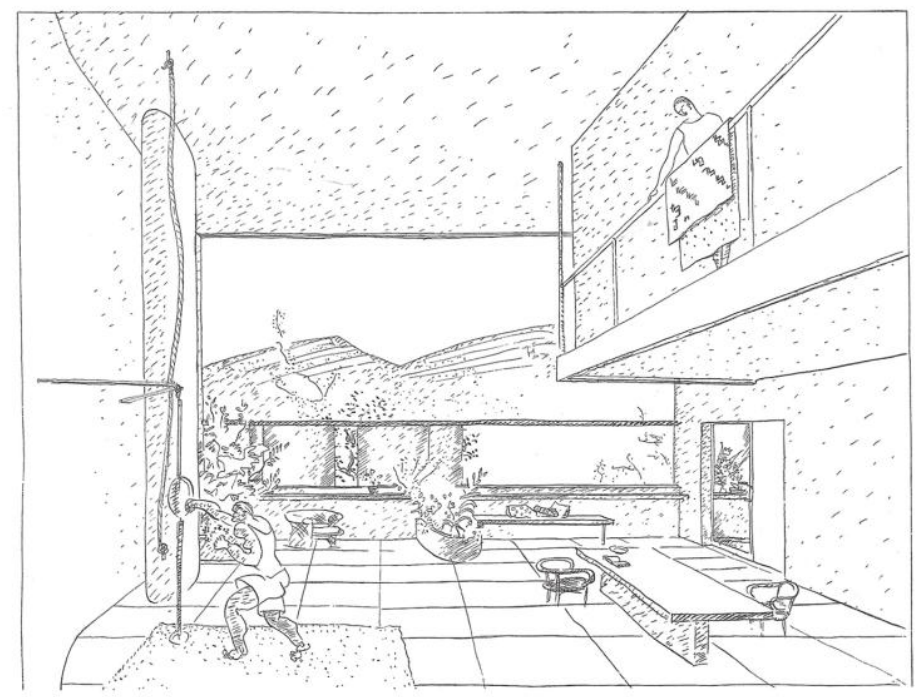

5. Projet Wanner, Genève 1928/29 in Oeuvre complète de Le Corbusier 1910-1929 @ FLC

Les esquisses du Corbu ouvraient et animaient le cadre du logis, en jouant des règles de la perspective pour mieux suggérer la continuité des espaces, en cadrant le paysage et en l'invitant à l'intérieur de l'habitat. L'insertion de petites scènes de vie dans ses croquis amplifiait la sensation de dépaysement et de détente: un boxeur s'entraînant au punching-ball sur une terrasse, un couple prenant un café dans la maison du contremaître, des enfants jouant sur le tapis du salon. L'esquisse est investie par Le Corbusier de plusieurs rôles : note et représentation, mais aussi pensée du projet par "la simulation de différentes solutions" comme l'envisage Gérard Monnier dans son article Esquisse de $1987{ }^{15}$. "Souvent délivrées sous la forme de messages personnels, les esquisses correspondent en fait au moment où l'investissement individuel de l'architecte est à son sommet, avant le développement du travail en agence ". Les personnages et actions qui y sont parfois croqués introduisent également une narration dans l'architecture projetée.

De même, les 'objets à réaction poétique' participent à l'espace, et des objets de la vie quotidienne figurent sur les photos de ses réalisations des années 20 , comme les cannes de golf posées dans le vestibule de la villa Savoye. A contrario des dessins 'habités', dans l'espace réel ce sont donc les objets qui suggèrent une présence non directement montrée. Ces artifices introduisent néanmoins la vie dans l'architecture, dès l'étape du projet. Ils participent au travail de communication, d'ailleurs considérable, de Le Corbusier et favorisent une projection du

15 'Esquisse' Gérard Monnier in Le Corbusier, une encyclopédie, Ed du Centre Georges Pompidou/CCI, Paris 1987. 
lecteur ou du visiteur dans ces espaces nouveaux, en écho à "l'investissement individuel de l'architecte " décelé par Gérard Monnier.

La villa Savoye semble conçue pour accueillir et réunir. Nous ignorons presque tout de la manière dont elle a été habitée, beaucoup pensent qu'elle n'est pas vraiment faite pour cela, et pourtant elle attire visiteurs et créateurs. C'est pourquoi la question des personnages est centrale dans mon projet de film. La perception engage les sens, l'esprit et l'imaginaire. Les thèmes induits par la villa se déclineront sur ces trois registres.

\subsection{L'approche documentaire}

Dans ce projet, la forme du film, indissociable du sujet, se doit d'être inventive. Tenter de refléter un regard architectural par une proposition cinématographique, en évitant l'analogie formelle directe. Pour répondre à la fluidité de l'espace, à la diversité des sensations et des perspectives dans la villa, à la transdisciplinarité et la recherche de synthèse artistique de Le Corbusier, le film cherche à incarner ces qualités plutôt que de les décrire.

A partir de mes souvenirs personnels et de mes photographies, j'ai cherché à retrouver mes sensations de découverte de la villa ; j'en ai parlé, j'y suis retournée, j'ai suivi une visite guidée. Assez rapidement j'ai cherché à rencontrer des interlocuteurs institutionnels, l'administrateur, la conservatrice, certains membres de la Fondation Le Corbusier. J'ai replongé dans les livres sur l'architecte, dans ses écrits, puis dans les archives. Je ne développerai pas ici toute cette partie de recherches documentaires, que vous connaissez. Parallèlement s'opère un travail de l'imaginaire, qui se nourrit de toutes ces données, certaines factuelles et d'autres plus psychologiques, afin d'entrevoir une direction pour le film.

En m'interrogeant sur la manière de filmer la villa Savoye, de ma mémoire de cinéphile surgissent quelques images fortes, associées à une ambiance, une couleur. Je revois les personnages hiératiques d'Alain Resnais dans L'année dernière à Marienbad (1961), déambulant dans un château et des jardins désertés, avec une gestuelle et un rythme hypnotisants. D'autres images reviennent, d'Antonioni ou de Tati. Parmi les artistes qui ont proposé des travaux dans la villa, la vidéo intitulée 'Singspiel', où Ulla von Brandenburg réunit en 2009 une 'famille' pour un repas dans le salon, m'avait marquée par sa force mais aussi une certaine familiarité avec mon propre projet, alors très embryonnaire. La sensation d'incommunicabilité et d'infinie répétition transmise par la chanson lancinante et une mise en scène montée en boucle hante l'esprit bien après son visionnage. J'y retrouve ma volonté d'investir vraiment le lieu, sans vouloir jouer sur cette corde de la mélancolie.

Je n'y ai pas pensé pendant tout le temps de l'écriture, mais je songe après coup au film Chronique d'un été réalisé par Edgar Morin et Jean Rouch en 1961. Les auteurs choisissent un panel de personnes de milieux différents, dont certaines parmi leurs amis, pour illustrer une diversité sociologique. Ils les filment dans des situations 'quotidiennes' mais préparées, un repas, une promenade dans la ville, pour tenter de faire émerger une expression sincère, naturelle. Ma démarche peut s'en rapprocher: créer des situations pour faciliter une libération de la parole et du geste, pour traduire une diversité de sensibilités à l'épreuve de la villa Savoye. À ce propos, Aline Caillet, dans son ouvrage sur les dispositifs, parle d' "expérience documentaire ": "le film, conçu comme une situation authentiquement vécue, les invite [les personnages-acteurs] à se produire, à s'inventer eux-mêmes dans le cadre posé." ${ }^{16}$. Pour atteindre à un niveau de profondeur, on doit renoncer à une improbable spontanéité. "Une personne, dès lors qu'elle est filmée, devient un personnage. Jouer son propre rôle, c'est encore -ou déjàen jouer un". ${ }^{17}$

\footnotetext{
${ }^{16}$ Caillet, Aline. Chapitre 2 :Renégocier la fiction dans le documentaire'in Dispositifs critiques, Rennes 2014.

${ }^{17}$ Ibid
} 
Comme la conception d'une architecture, celle d'un film s'élabore au fil d'allers et retours entre le premier dessein et les contraintes du réel, les intentions et le possible, les lieux et leur accessibilité, les protagonistes, leur disponibilité et l'histoire qui peut se tisser avec eux et entre eux. Ce que j'expose ici de mon projet aurait pu se comparer à un Avant-Projet Définitif en architecture, assez avancé dans sa définition. Mais l'incertitude de sa production, en particulier celle d'une date de tournage conditionnée par un financement difficile, me pousse à le présenter comme un 'Work in progress'. Pour cette même raison, je ne ferai pas une description précise du traitement du film. J'en présente plutôt quelques caractéristiques en lien avec le sujet.

\subsection{Recherche de personnages}

"Pour donner chair à ses discours L.C. convoque une galerie de portraits, caricatures, êtres de fable. Il en brosse des tableaux simplistes. Il sait à la perfection utiliser à fin de démonstration des petits morceaux d'humanité qualifiés, stylisés par le rôle qu'il entend leur faire tenir" ${ }^{18}$ remarque Marie-Christine Loriers.

'Mes' personnages seront des êtres de chair, invités à jouer leur propre rôle dans des situations tout à fait réalistes au regard de leur activité. La difficulté, et le travail d'auteur de ce point de vue, consiste à favoriser des rencontres, puis de construire un cheminement vivant et humain à travers le film et dans la villa.

Dans ce but je choisis ces 'invités' selon des critères assez inhabituels pour un documentaire. Avant tout ils ont un rapport personnel avec la villa Savoye, au-delà d'un lien avec le lieu ou avec l'architecture par leur activité professionnelle, ce qui favorisera une lecture plurielle, des approches expérimentales, voire émotionelles. Gardien, chorégraphe ou petit-fils des habitants, ils pourront se passer le témoin en croisant des thématiques ou des souvenirs communs. Chacun d'entre eux est donc capable de se relier à un ou plusieurs autres invités. La question centrale du projet étant celle de notre perception actuelle de la villa Savoye, les invités s'exprimeront par le corps, la gestuelle, la parole, un crayon, le rythme, le regard, un texte poétique, un souvenir. Enfin ces choix sont indépendants de la renommée ou de la reconnaissance scientifique.

Ma méthode a donc consisté à définir des rôles possibles, une sorte de casting idéal à partir duquel j'ai recherché des personnes coïncidant avec ces attentes. Cette quête est longue, mais passionnante quand les personnes rencontrées incarnent une des facettes envisagées. Cela devient enthousiasmant quand la personnalité de l'invité appporte bien plus que ce que j'avais imaginé. Contacts, rencontres répétées, si possible dans des contextes de travail, le temps est nécessaire pour les écouter, les connaître. Les paroles entendues, les attitudes, les situations observées vont constituer le matériau avec lequel s'écrira plus précisément le film. Mais avant d'aborder cette étape, je vais présenter deux des rôles choisis pour illustrer mon propos.

\subsubsection{Les passeurs : regard, parole, dessin}

La question de la transmission s'incarne idéalement avec des étudiants en architecture. Par leur situation intermédiaire, à mi-chemin entre un public néophyte et des professionnels de l'architecture, les étudiants peuvent jouer le rôle de passeurs. Comment interrogent-il un de leurs professeurs, quel regard portent-ils? Sont-ils curieux, indifférents ou en opposition à cette architecture qui aura bientôt un siècle? qu'en font-ils ? Ils représentent à la fois la découverte et l'élaboration d'un futur.

Je dirige rapidement mes recherches vers l'Ecole Nationale Supérieure d'Architecture de Paris-Belleville qui a hébergé pendant des décennies le groupe Uno, fondé par Henri Ciriani, Jean-Patrick Fortin, Edith Girard et Claude Vié. Ces architectes avaient élaboré à la fin des années 1970 une méthode d'enseignement dite 'verticale'

\footnotetext{
${ }^{18}$ Loriers, Marie-Christine : L'icône et le locuteur, p14, in Corbu vu par, ouvrage collectif 1987
} 
car pensée dans la continuité, pour accompagner les étudiants au fil des ans, et s'appuyant sur les apports de l'architecture moderne, particulièrement celle de Corbu. Il reste aujourd'hui peu d'enseignants de ce groupe. L'un d'entre eux, Alain Dervieux, s'est entièrement consacré à l'enseignement. Il communique facilement son énergie et son enthousiasme. Son axe de travail est 'le visible'. Très à l'écoute de ses étudiants, il les fait réfléchir par la pratique sur la perception visuelle qu'aura un habitant ou un visiteur dans les espaces qu'ils conçoivent, les invite en permanence à se mettre à la place de l'usager. Tout en les incitant à l'expérimentation, il leur fournit des outils de conception et d'analyse. Il considère l'architecture moderne, et en particulier la villa Savoye, comme une ressource pédagogique permanente.

Il propose ainsi à ses étudiants un exercice appelé le '30x30' inspiré par le carré de 20 mètres sur 20 de la villa. Sur le thème du plan libre, il leur demande d'aménager l'espace et d'apporter la lumière dans une bibliothèque, structure carrée portée par une trame de poteaux, en respectant certaines règles. Avec un préalable déterminant : le choix d'une 'hypothèse'. Chacun décide d'une notion qu'il veut investir. Il l'exprime par un mot, puis l'analyse afin de la décliner en trois temps qui lui permettront de la traduire spatialement, tout au long de l'exercice. 'Effervescence', 'nébuleuse', 'pluie d'or', 'envie'... les hypothèses sont le support d'une réflexion sensible sur l'espace. Ce choix fait, la première étape porte sur l'entrée: dessiner un poteau, son rapport à la dalle qu'il supporte, son jeu avec la lumière naturelle. Ce travail très élémentaire, qui part de presque rien, soulève déjà beaucoup de questions. Sa méthode progressive me semble idéale pour aider le spectateur à aborder quelques notions fondamentales d'architecture. J'ai suivi le travail du studio depuis deux ans, faisant connaissance avec les étudiants, sondant leurs préoccupations, observant leurs attitudes. Il existe des contrastes forts entre eux, quelques passionnés présentent spontanément leurs dessins et photos de la villa Savoye et d'autres bâtiments visités, certains plus indifférents se contentent de découvrir par le truchement d'Internet, mais je note toujours une dynamique d'ouverture. Je poursuivrai cette observation si possible jusqu'au moment du tournage, afin d'être suffisamment proche d'eux pour pouvoir recueillir une parole encore hésitante. En attente de financement, je dois tenir le projet 'prêt au tournage' et maintenir ces contacts essentiels. Je choisirai pour le film quelques étudiants qui s'expriment assez facilement par le dessin ou la parole. Je capterai les échanges avec leur enseignant avant de les filmer dans la villa, interrogeant, dessinant.
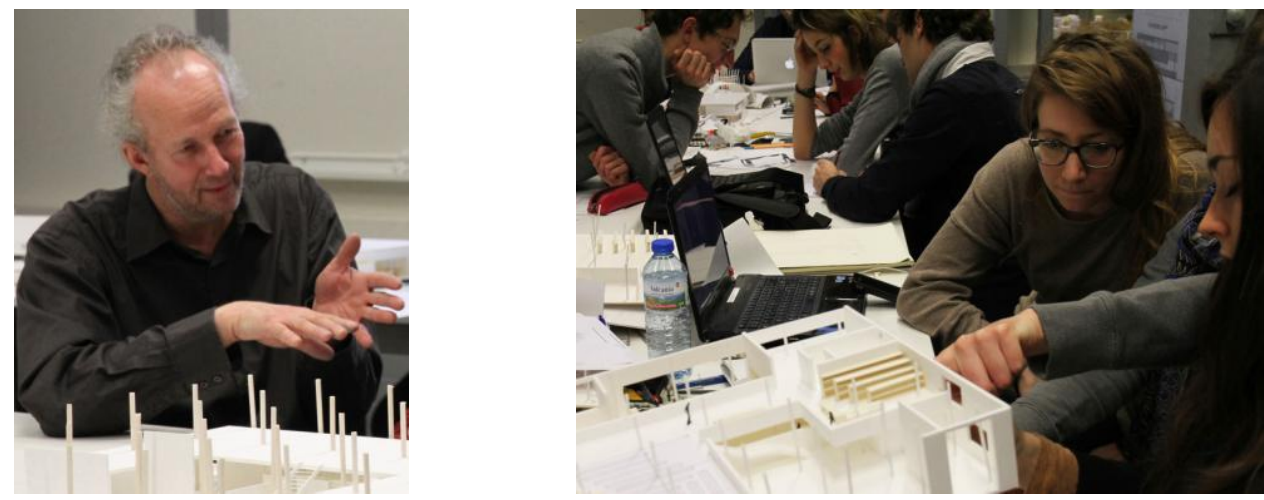

6. - 7. Alain Dervieux et quelques étudiants du studio 30x30, ENSA Paris-Belleville, 2014.

Assez rapidement, j'envisage l'intervention d'Edith Girard, architecte co-fondatrice du groupe Uno qui s'est un peu éloignée d'une certaine primauté de la forme. Parallèlement à son activité d'enseignante, elle a construit des logements collectifs avec beaucoup d'engagement, défendant une qualité des espaces individuels et communs. Dès la première rencontre je suis conquise par cette forte personnalité, volontaire et généreuse. Elle m’explique qu'au début de ses études elle n'était pas très sensible à l'architecture de Le Corbusier, et qu'elle devient de plus en plus 'fan' au fil des ans. Elle évoque la pédagogie du groupe Uno, basée sur le collectif, l'importance de 
notions telles que l'espace ouvert, la continuité, l'expérimentation, la place du dessin et de la maquette, du rapport direct à l'espace en l'arpentant. J'écris quelques situations du film où elle aura toute sa place. En assistant à plusieurs échanges entre elle et Alain Dervieux (qui fut un de ses premiers étudiants), argumentés et vivants, je sais qu'ils pourront proposer des points de vue clairs même pour un spectateur néophyte.

Malheureusement Edith Girard est décédée prématurément, en septembre 2014. Ce projet lui est dédié.

\subsubsection{Les explorateurs : corps et mouvement}

Quand j'ai commencé à travailler sur ce film, des scènes 'rêvées' ont rapidement traversé mon esprit. L'une des premières était une chorégraphie dans la villa Savoye. Comment mieux interroger notre perception sensorielle de la villa que par l'expérimentation du corps?

La présence de la danse est primordiale dans ce documentaire. Parce que le rapport au corps était une des préoccupations majeures de Le Corbusier quand il concevait une architecture. Parce que la promenade architecturale et la plastique de la villa y invitent. Et pour répondre à sa propre proposition dans le film Architecture d'aujourd'hui dont j'ai parlé plus tôt. Edith Girard m'a aidée dans ma recherche en évoquant un atelier proposé à ses étudiants dans la villa Savoye par la chorégraphe Daria Faïn, à la fin des années 80. J'ai retrouvé sa trace et l'ai rencontrée. Son travail porte depuis longtemps sur le rapport corps-architecture. Intéressée par le projet du film, elle est prête à reprendre l'idée de l'atelier. Nous sommes allées ensemble à la villa Savoye, où elle n'était pas retournée depuis presque trente ans. Lui sont revenus des souvenirs de postures des étudiants, à qui elle avait proposé de prendre leur corps comme mesure pour faire un relevé de l'architecture, en s'y collant, et d'en déceler les rythmes pour les reproduire. Depuis nous avons entamé un dialogue à distance, puisqu'elle vit aux Etats-Unis, pour préparer sa participation au film, dont une partie sera chorégraphiée.
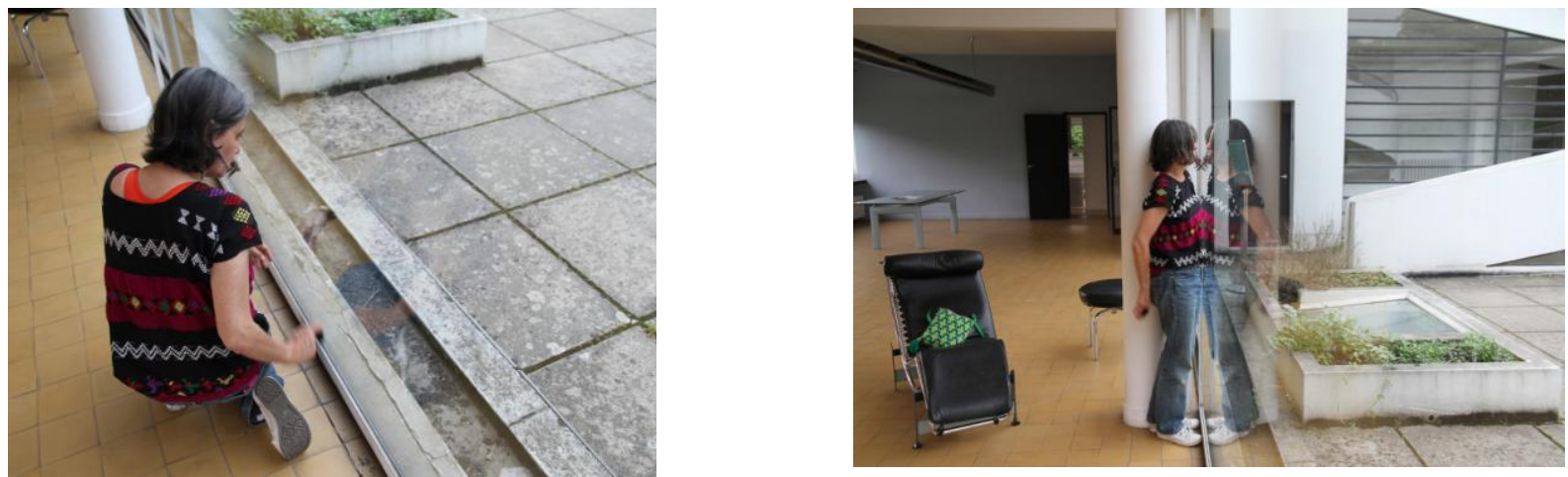

8. - 9. La chorégraphe Daria Faïn revenant dans la villa Savoye, plus de vingt ans après l'atelier qu'elle y a animé auprès d'étudiants en architecture. (C) FLC (C) A.D.

\subsection{Se confronter au lieu}

Chaque invité, par son activité et sa sensibilité, peut participer dans le film à plusieurs actions et propositions thématiques, qui s'articulent en deux ou trois situations. Quel est le lieu le plus à même d'accueillir chacune d'elles, de la faire résonner ? Et réciproquement quelle situation servira telle qualité d'une pièce ? Tout le travail de préparation du film, repérages et recherches, aboutit à une forme de connaissance des lieux comme des invités qui permet de poser une liste d'hypothèses, qu'il faut ensuite affiner par les échanges et l'écriture. Je m'interroge aussi sur les croisements, rencontres susceptibles d'éclairer ces situations, de les relier. Guidée par la nécessité de tracer un parcours filmique, avec ses accidents et ses plages de contemplation, je dresse une sorte de 
cartographie. C'est un exercice de synthèse qui passe par des phases de confusion. Une première écriture du film apparaît, comme une sorte de puzzle dont chaque pièce a plusieurs contours possibles.

Un travail de mise en scène démarre. Sans jamais oublier qu'il s'agit d'un documentaire : écrire une trame assez serrée pour construire un récit, assez lâche pour permettre au réel de s'y développer. En effet, si je peux proposer des configurations aux invités, ce sont eux ensuite qui s'expriment et agissent. Les liens multiples entre eux permettront également une liberté au montage, tenant compte du développement imprévisible de chaque 'scène'. Une recomposition libre, faisant écho à l'idée d'un plan libre. De même la fluidité de l'espace de la villa imprimera celle du film : le passage d'un thème à un autre, d'une situation à une autre se fera dans la suggestion, un glissement, un regard. Si la forme finale du film est forcément unique, linéaire, comme l'un des parcours possibles dans la villa, elle tentera de rendre perceptibles les ouvertures, d'ouvrir des chemins à l'imaginaire.

Pour le tournage, il faut également préciser la manière de filmer. Les cadrages sont importants ; on sait que la villa en propose certains que nous connaissons par de nombreuses photographies. Sans les renier, il serait intéressant d'aller plus loin dans l'observation des formes et de l'espace, tant pour les plans montrant l'architecture 'nue' que pour les plans habités. Si la question de l'usage n'est plus dans la villa celle de l'habiter, il n'en est pas moins important d'observer les corps, de capter leurs contacts avec les éléments, les sons produits, une main qui effleure une surface, un coude qui s'appuie sur une allège, le rythme du pas pour monter la rampe, etc. Les situations sont également proposées avec cette préoccupation.

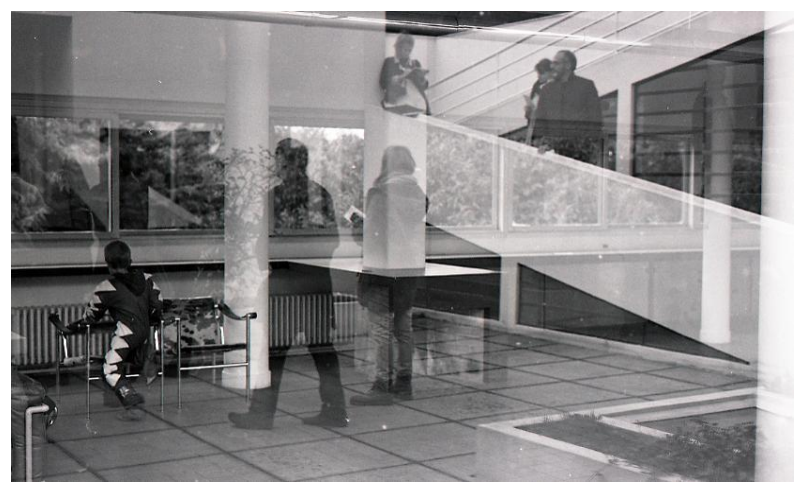

10. Photographie de C.Lefranc, étudiant du studio 30x30, ENSA Paris-Belleville, 2013.

\subsubsection{Un exemple de situation à partir d'un lieu : le hall d'entrée}

Le hall est un espace d'accueil, vaste, où sont disposés librement des éléments hétérogènes; l'escalier, spirale maçonnée qui s'élève verticalement, s'oppose à l'oblique de la rampe, et à l'horizontale dessinée par la courbe vitrée de l'enveloppe. Chaque élément y est distinct, jusqu'au lavabo qui trône derrière l'un des pilotis, et mis en valeur par les rais de lumière arrivant de part et d'autre. Le hall, véritable proposition plastique, semble un lieu idéal pour déclencher la chorégraphie.

Daria Faïn y explore les formes et les proportions, les matières. Elle peut jouer avec les reflets ou se montrer par la transparence de la vitre aux étudiants qui arrivent du parc et découvrent la villa. Elle peut y croiser l'itinéraire d'un autre invité, leurs déplacements exacerbant l'opposition entre l'escalier et la rampe. Elle arpente son plan incliné et souligne le dessin des ouvertures. Elle traverse un petit groupe de visiteurs arrêté sur le palier intermédiaire, écoutant la lecture d'une lettre de Madame Savoye adressée à Le Corbusier et se plaignant des fuites d'eau dans la maison. 


\section{Apports de l'écriture filmique}

La plongée dans l'écriture de ce film documentaire sur la villa Savoye m'a permis de la revisiter d'une manière différente de ma première approche d'architecte, mais cependant connectée à elle. D'abord parce que le documentaire implique un travail de recherche préalable, se nourrit de son sujet pour trouver une forme pertinente en accord avec son propos, ensuite parce que de nombreux thèmes rapprochent les deux disciplines que sont l'architecture et le cinéma. Ma lecture de la villa s'est donc enrichie de ce travail 'sur le terrain', à la rencontre de l'objet et de ceux qui l'observent selon différents points de vue. Le film ouvre à d'autres modes d'expression que le texte et la photo, et les englobe : voix, sons, mouvements, séquences de vie. Ces croisements favorisent un approfondissement de la réflexion formelle en reliant observation et imaginaire, et en s'appuyant en permanence sur la question de la perception.

Pour ce qui est du projet de film, les dossiers de présentation n'ont pas permis d'obtenir des aides financières. La forme choisie, qui n'est ni classique ni dans le style 'télé' dominant aujourd'hui, et le fait que l'architecture fait peur aux diffuseurs, ont rendu ces démarches inefficaces dans un premier temps. J'ai alors décidé de formaliser assez précisément mes intentions pour aboutir à un scénario, forcément virtuel, mais révélant concrètement le dispositif et les choix de réalisation. Ce 'traitement' a séduit un producteur auparavant rétif au sujet. Et plusieurs professionels du documentaire, après l'avoir lu, m'ont dit avoir eu pour la première fois l'impression de comprendre un peu ce qu'est l'architecture. En poursuivant dans cette voie, en cherchant une présentation adaptée et des appuis moins conventionnels, je compte débloquer cet obstacle financier pour commencer le tournage bientôt. Il s'agit donc d'un processus en cours, qui parle de la transmission de l'architecture aujourd'hui, et de ses difficultés.

Comme je l'ai précisé, j'ai été amenée à écrire une sorte de scénario pour convaincre des lecteurs, néophytes en architecture, que ce sujet pouvait être accessible voire intéressant, et que le dispositif choisi était non seulement légitime mais aussi cinématographique. J'ai trouvé deux autres avantages à cette étape inhabituelle et très instructive. D'abord c'était ma première expérience d'écriture aussi proche de la fiction. Au-delà du plaisir que j’y ai trouvé, j'ai pu vérifier la cohérence des choix de personnages et de thématiques, manifeste en particulier quand je devais intervertir deux séquences, privilégier un enchainement plutôt qu'un autre. J'expérimentais alors la fluidité, la variété des parcours possibles dans la construction du film pour provoquer des sensations différentes. Cet exercice était comme une première validation par la maquette de la structure du film mais aussi de son adéquation avec le sujet. Ensuite cela m'amène à reconsidérer la fiction comme un autre mode de médiation de l'architecture. Réflexion et expérimentation à poursuivre.

\section{Conclusion}

J'ai exposé une approche documentaire parmi beaucoup d'autres existantes ou à inventer. Lors de cette courte présentation j'espère avoir montré que l'écriture filmique offre à l'architecture un champ d'exploration. Elle crée un rapport différent au sujet d'étude. Le film embarque son auteur sur le site, et peut montrer des évènements autant que des formes et des discours. Exploration formelle, le film permet de parcourir une architecture, de la voir sous divers aspects, dans la durée, de l'habiter, la confronter à différents visiteurs. Le film pose sans cesse la question du regard, du point de vue, et de la réception. Il permet aussi d'intégrer une dimension poétique à l'apport d'informations, de jouer avec les frontières de la fiction pour mieux révéler et pour toucher le spectateur.

C'est un mode d'investigation autant que de représentation. 
Je ne peux résister au plaisir de citer Le Corbusier parlant de la caméra, dans son texte Esprit de vérité de 1933: "Je dis alors que l'objectif sans nerfs ni âme est un voyeur prodigieux, un découvreur, un révélateur, un proclamateur. Et par lui nous pourrons entrer dans la vérité de la conscience humaine. Le drame humain nous est ouvert. C'est cela qui nous intéresse." ${ }^{\prime 19}$.

J'ai tenté dans cette brève présentation de montrer l'intérêt du documentaire pour l'architecture. Plus largement, je pense qu'une pratique et une réflexion sur le cinéma, documentaire et fiction, fondées sur la réalisation et l'analyse de films, ont une légitimité dans l'enseignement et la recherche en architecture. Mode de médiation, de sensibilisation, le film et les questions qu'il pose peuvent devenir outils de connaissance, et offrent de vraies pistes de réflexion aux architectes.

Je termine par l'exposition du début du film:

\section{Dans un garage - nuit}

nouveau dans la clairière où se dresse la silhouette étrange de la villa, dont il commence à faire le tour...

Un homme dort dans un sac de couchage; à côté de lui, un chien.

Un réveil sonne, l'homme allume une lampe-torche, vérifie l'heure, regarde autour de lui : un espace vide, une sorte de petit hangar.

Il se lève, met une veste et ouvre une porte métallique : "allez Hector, on y va”. Hector bondit dehors.

L'homme, gardien de nuit, éclaire ici et là avec sa lampe torche le bâtiment dont il sort : la villa Savoye. Il s'amuse en créant des jeux d'ombre sur les façades et les pilotis, puis sur les arbres environnants.

Il s'éloigne et entame sa ronde dans le parc. Dans l'obscurité animée par les mouvements de sa main tenant la lampe, on entend ses pas, on devine sa silhouette, les cailloux d'un chemin, feuilles, branches, la silhouette d'un mur de clôture qui se prolonge par une grille. Puis face à lui, à l'entrée du parc, apparaît la loge du jardinier, toute petite maison perchée sur deux pilotis. Il soupire "j'aimais mieux dormir là !".

Le gardien observe, écoute. Il tourne vers sa gauche et s'engage dans un bosquet d'arbres. Il débouche à

\section{Remerciements}

Je tiens à remercier pour leur gentillesse et leur disponibilité les membres de la Fondation Le Corbusier, et en particulier Michel Richard, Directeur de la Fondation, pour son accueil attentif ainsi qu'Arnaud Dercelles, responsable du Centre de documentation et de recherche, pour son aide avisée.

Je remercie également les équipes administrative et enseignante de l'ENSA Paris-Belleville, pour leur accueil chaleureux et leur soutien. Je remercie notamment Alain Dervieux, architecte-enseignant, pour sa générosité et son implication.

\section{Bibliographie}

Caillet, Aline: Dispositifs critiques. Le documentaire, du cinéma aux arts visuels. Rennes: Presses universitaires de Rennes, Collection Arts contemporains, 2014.

François, Arnaud : La cinématographie de l'oeuvre de Le Corbusier, in Revue Cinémathèque, printemps 1996.

Le Corbusier : Esprit de vérité in la revue Mouvement n² 1 , Paris, juin 1933.

\footnotetext{
${ }^{19}$ Le Corbusier : Esprit de vérité in Mouvement, Paris, 1933.
} 
Le Corbusier : Vers une architecture, Paris 1923.

Loriers, Marie-Christine and others: Corbu vu par, ouvrage collectif. Liège : Pierre Mardaga Editeur, 1987.

Monnier, Gérard : Esquisse in Le Corbusier, une encyclopédie. Paris : Edition Centre Georges Pompidou/CCI, 1987.

Pancher, Annick : Existe-t-il un espace public télévisuel pour l'architecture contemporaine ? Directrice : Hélène Puiseux. D.E.A. Cinéma, Télévision, Audiovisuel. E.P.H.E. et Université Paris1 Panthéon-Sorbonne, UFR Histoire de l'Art et Archéologie, 1999.

Prelorenzo, Claude: Quand Corbu faisait son cinéma in Le Visiteur $\mathrm{N}^{\circ} 17$. Paris : Société française des architectes, 2011.

Simond, Clotilde and others: In situ - de visu - in motu, Architecture, cinéma et arts technologiques. Gollion : Infolio éditions, 2014. 